CASE REPORT
A.M.G. Gonçalves
P. Page
V. Domigo
J.-F. Méder
C. Oppenheim

\section{Abrupt Regression of a Meningioma after Discontinuation of Cyproterone Treatment}

SUMMARY: The multiplicity of meningiomas or abrupt lesion growth in patients treated with cyproterone acetate suggests that this progestative treatment may promote lesion growth. We report the rapid regression of an incidental meningioma after discontinuation of a 10-year cyproterone acetate treatment. This unique observation suggests that conservative management of meningiomas may be the best option among users of high doses of cyproterone acetate, given that spontaneous regression may occur after hormonal treatment discontinuation.

ABBREVIATIONS: FLAIR = fluid-attenuated inversion recovery

C yproterone acetate is a synthetic progestin with a powerful antiandrogen property, which is indicated for the treatment of inoperable prostate carcinoma, the reduction of sexual drive in men, or severe signs of androgenization in women. It is occasionally prescribed in male-to-female transsexual patients. It is also found in smaller quantities in combined oral contraceptives and for treating seborrhea, acne, hirsutism, and moderate androgen-related alopecia. Recent observations of meningioma, often multiple, among users of high doses of cyproterone acetate have raised the suspicion that it may promote the rapid growth of pre-existing or new meningioma. ${ }^{1}$ Here, we report the rapid regression of a meningioma after discontinuation of a 10-year cyproterone acetate treatment. This unique observation strengthens the link between cyproterone acetate and intracranial meningioma.

\section{Case Report}

A 46-year-old woman was admitted to the stroke unit of our institution for sudden transient neurologic symptoms, including left upper limb paresthesia. Her medical history was unremarkable. During the previous 10 years, she had been taking oral cyproterone acetate (50 $\mathrm{mg} /$ day), which acts as a progesterone agonist, in combination with substitutive estradiol, for androgenetic alopecia. Brain MR imaging, performed on admission, showed 2 punctuate cortical acute ischemic strokes (not illustrated) in the right superficial middle cerebral artery territory, corroborating the acute neurologic presentation. A right frontal extracranial tumor was also found incidentally (Fig 1). The tumor presented as a dural-based mass with cortical buckling and a thin rim of trapped CSF. It appeared as an isointense mass on the T1-weighted sequence, slightly hyperintense on the FLAIR sequence, with homogeneous contrast enhancement on a postgadolinium 3D T1-weighted sequence, and surrounded by a rim of peritumoral vasogenic edema on the FLAIR sequence. These imaging characteristics strongly favored the diagnosis of a frontal meningioma. The etiologic vascular work-up was negative apart from a patent foramen ovale. Because of its inherent thromboembolic risk, the hormonal treatment was discontinued, and the patient was discharged from the hospital under low-dose antiplatelets. On a serial MR imaging follow-up, the

Received August 13, 2009; accepted August 17.

From the Departments of Neuroradiology (A.M.G.G., C.O., J.-F.M.), Neurosurgery (P.P.), and Neurology (V.D.), Université Paris Descartes, Centre Hospitalier Sainte-Anne, Paris, France.

Please address correspondence to Catherine Oppenheim, MD, PhD, Department of Neuroradiology, Université Paris Descartes, Centre Hospitalier Sainte-Anne, 1 rue Cabanis, 75674 Paris Cedex 14, France; e-mail: c.oppenheim@ch-sainte-anne.fr

DOI 10.3174/ajnr.A1978 frontal mass had unexpectedly shrunk $\left(16 \mathrm{~cm}^{3}\right.$ at admission, $3.1 \mathrm{~cm}^{3}$ at 3 months, and $1.9 \mathrm{~cm}^{3}$ [88\% volume reduction] at 6 months) and the peritumoral edema had disappeared. The patient was free of symptoms.

\section{Discussion}

A meningioma is a benign tumor usually arising from the arachnoid cap cells or meningothelial cells. The relationship between meningioma and sex hormones is well known, with a higher female-to-male ratio, especially during the female reproductive years. Meningiomas may enlarge during hormonal influx, particularly during pregnancy and in the luteal phase of the menstrual cycle. ${ }^{2}$ In line with this, spontaneous regression of meningiomas has been reported after pregnancy. ${ }^{3}$ Because meningiomas often express progesterone receptors and, albeit to a lesser extent, estrogen receptors, ${ }^{4,5}$ it has been postulated that these hormones may influence the increase in tumor size by increasing blood volume and extracellular and intracellular fluid as well as by increasing proliferation of the tumor tissue.

As with breast cancer, exogenous female steroid hormones, especially progestogens, may play a role in the occurrence of meningioma. ${ }^{6,7}$ The risk of meningioma is increased among postmenopausal women who have used hormonal treatment replacement therapy and among women who have used longacting contraceptives. ${ }^{7}$ The suspicion that high daily doses of cyproterone acetate for long periods could be responsible for the development of meningiomas, and especially multiple meningiomas, was reported in $2008 .{ }^{1}$ Froelich et $\mathrm{al}^{1}$ reported multiple meningiomas in 9 female patients treated with cyproterone acetate. Before treatment withdrawal, an increase in tumor size and/or the development of new meningiomas was observed in all 6 patients with available imaging follow-up. Treatment withdrawal was associated with lesion size/number stabilization. In 2007, the abrupt growth of a meningioma was reported in a male-to-female transsexual patient who had been taking high doses $\left(100 \mathrm{mg}\right.$ ) of cyproterone acetate. ${ }^{8}$ Despite evidence that antiprogesterone treatment reduces the size of meningioma, both in vitro and in vivo, such hormonotherapy is not part of the conservative management of meningioma. ${ }^{9}$

Reports of spontaneous regression of meningiomas in the literature are rare. Apart from spontaneous resolution after intratumoral hemorrhage, ${ }^{10}$ only 2 cases of regression have been reported in association with progestative hormonal treatment withdrawal. In a female case of lymphangio- 

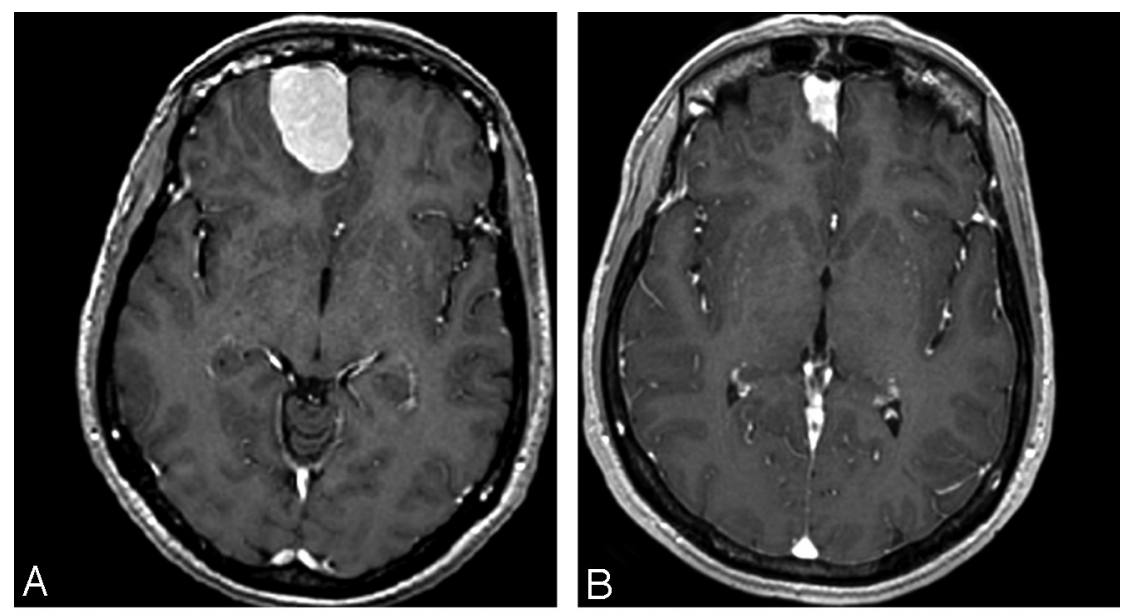

Fig 1. Extra-axial lesion of the olfactory groove, homogeneously enhanced on axial and T1-weighted sequences after administration of gadolinium and reformatted in the axial $(A$ and $B$ ) and sagittal $(C$ and $D$ ) planes. The lesion volume decreased rapidly from $16 \mathrm{~cm}^{3}(A$ and $C)$ on admission to 3 $\mathrm{cm}^{3}$ ( $81 \%$ reduction in volume) at 3 months (not shown) and to $1.9 \mathrm{~cm}^{3}$ ( $88 \%$ reduction in volume) ( $B$ and $\left.D\right)$ at 6 months after hormonal treatment withdrawal.
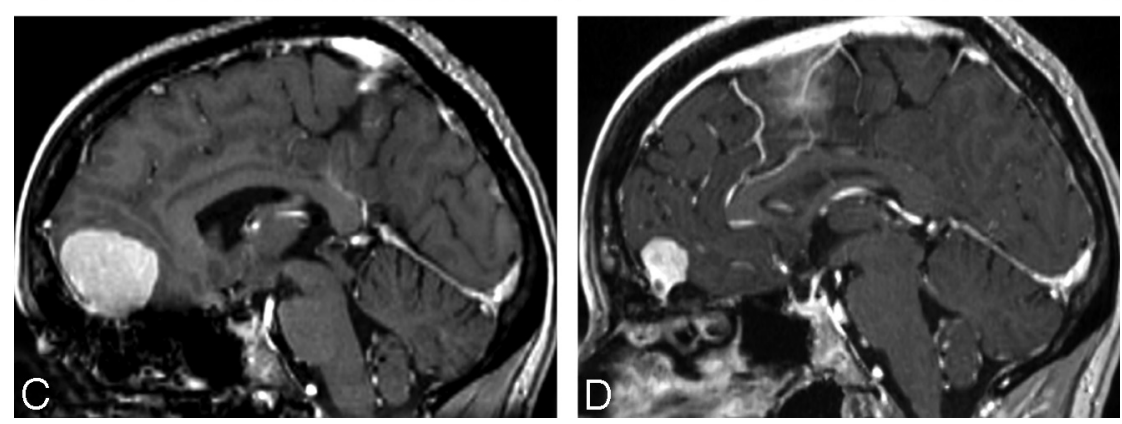

leiomyomatosis, the growth of multiple meningiomas was observed during a 4-year period after administration of medroxyprogesterone acetate, followed by a spontaneous regression after hormonal withdrawal. ${ }^{11}$ Shimizu et $\mathrm{al}^{12}$ reported a similar regression of meningioma during a 2-year period in an 80-year-old man after suspension of progesterone agonist therapy, administered for benign prostatic hyperplasia. ${ }^{12}$ No previous cases of meningioma regression have been observed after discontinuation of treatment with cyproterone acetate.

Our observation of rapid meningioma regression after hormonal treatment discontinuation reinforces the link between meningioma and cyproterone acetate. Lesion-volume regression might be due to a rapid decrease of tumor growth induced by steroid receptors. ${ }^{13}$ We cannot formally exclude the possibility that estradiol discontinuation may have played a role in the lesion regression. Any such role is likely to have been minor, given that the patient was taking a low dose of substitutive estrogen therapy to counterbalance the antiestrogenic effect of cyproterone. Furthermore, estrogen receptors are often minimally present or absent in meningioma.

Taken together, the multiplicity of meningiomas and rapid lesion growth in patients treated with cyproterone acetate and, as shown here, the rapid lesion regression following treatment withdrawal suggest that this treatment may actively stimulate, and its withdrawal inhibit, the meningioma cell cycle. The link between meningioma and cyproterone acetate may be stronger than that with other progestative therapies. If confirmed, this may have several practical implications: 1) Systematic screening for meningiomas may be useful before cyproterone therapy is considered. 2) The hormonal history should be an- alyzed in all patients with meningioma. 3) If a meningioma is found with the patient under cyproterone acetate medication, conservative tumor management with serial MR imaging follow-up could be an option, given that regression may occur after treatment discontinuation.

\section{References}

1. Froelich S, Dali-Youcef N, Boyer P, et al. Does cyproterone acetate promote multiple meningiomas? In: Proceedings of the 10th European Congress of Endocrinology, Berlin, Germany. May 3-7, 2008

2. Wigertz A, Lonn S, Hall $\mathrm{P}$, et al. Reproductive factors and risk of meningioma and glioma. Cancer Epidemiol Biomarkers Prev 2008;17:2663-70

3. Smith JS, Quinones-Hinojosa A, Harmon-Smith M, et al. Sex steroid and growth factor profile of a meningioma associated with pregnancy. $\mathrm{Can} \mathrm{J} \mathrm{Neu}$ rol Sci 2005;32:122-27

4. Blankenstein MA, Verheijen FM, Jacobs JM, et al. Occurrence, regulation, and significance of progesterone receptors in human meningioma. Steroids 2000;65:795-800

5. Hatiboglu MA, Cosar M, Iplikcioglu AC, et al. Sex steroid and epidermal growth factor profile of giant meningiomas associated with pregnancy. Surg Neurol 2008;69:356-62

6. Moss J, DeCastro R, Patronas NJ, et al. Meningiomas in lymphangioleiomyomatosis. JAMA 2001;286:1879-81

7. Wigertz A, Lonn S, Mathiesen T, et al. Risk of brain tumors associated with exposure to exogenous female sex hormones. Am J Epidemiol 2006;164:629-36

8. Gazzeri R, Galarza M, Gazzeri G. Growth of a meningioma in a transsexual patient after estrogen-progestin therapy. N Engl J Med 2007;357:2411-12

9. Matsuda Y, Kawamoto K, Kiya K, et al. Antitumor effects of antiprogesterones on human meningioma cells in vitro and in vivo. J Neurosurg 1994;80:527-34

10. de Almeida JP, Petteys RJ, Sciubba DM, et al. Regression of intracranial meningioma following intratumoral hemorrhage. J Clin Neurosci 2009;16:1246-49

11. Pozzati E, Zucchelli M, Schiavina M, et al. Rapid growth and regression of intracranial meningiomas in lymphangioleiomyomatosis: case report. Surg Neurol 2007;68:671-74

12. Shimizu J, Matsumoto M, Yamazaki E, et al. Spontaneous regression of an asymptomatic meningioma associated with discontinuation of progesterone agonist administration. Neurol Med Chir (Tokyo) 2008;48:227-30

13. Jay JR, MacLaughlin DT, Riley KR, et al. Modulation of meningioma cell growth by sex steroid hormones in vitro. J Neurosurg 1985;62:757-62 